\section{A forced-choice study of edge detectors in the human visual system*}

\author{
STANLEY WOLL $\dagger$, CHARLES W. ERIKSEN, and HAROLD W. HAKE. \\ University of Illinois, Urbana, Illinois 61820
}

Psychophysical studies using masking procedures to investigate the possible existence of edge detectors in the human visual system have typically been flawed by nethodological errors and omissions that make their findings uninterpretable. An :xample of such a study is given and evaluated, and a replication of that study, using a ipatial forced-choice methodology, is reported in detail. The results of this replication are hown to contradict the critical findings of the earlier study; furthermore, it is suggested hat any ambiguities in the results of this replication can be interpreted in terms of nethodological and procedural factors, rather than in terms of higher-order neurological zonstructs. Finally, a further test of this methodological interpretation is proposed.

Following the recent success of nicroelectrode research on single-cell feature analyzers in lower animals, a number of psychophysical studies have been directed toward the question of whether such specific analyzing mechanisms might also exist in the human visual system (see Weisstein, 1969, for a review of the literature in both areas).

One particularly compelling neurophysiological finding along these lines is the discovery by Hubel and Wiesel $(1959,1968)$ of single-cell edge detectors in the visual systems of the cat and monkey. Such a finding has important implications for the area of human pattern recognition (cf. Neisser, 1967) and, thus, has stimulated a number of psychophysical studies at the human level. Included among these analogical studies are two experiments by Gilinsky $(1967,1968)$, which employed a forward masking procedure to examine the role of line detectors in human vision.

Gilinsky's experimental procedure can be summarized briefly as follows: ${ }^{1}$ The basic task required discrimination of the orientation of a striated test pattern after inspection for a given period of an adapting pattern of the same or opposite (perpendicular) orientation. The adapting durations ranged from 0.1 to $8 \mathrm{sec}$, and the duration of the test grating was set at a $50 \%$ threshold level for each $S$ (i.e., that duration at which the orientation of the test grating was correctly identified $50 \%$ of the time when viewed following a nonstriate adapting flash of the same luminance as the striated adapting pattern).

*This investigation was supported by United States Public Health Service Research Grant MH-1206 and United States Public Health Service Research Career Program Award K6-MH-22,014.

†This study represents part of a thesis submitted by the first author to the University of Illinois in partial fulfillment of the requirements for an MA degree.
There was no time interval between the presentations of the two patterns, except for the onset time of the second, or test, field. "Catch trials," consisting of the presentation of a blank or homogeneous test field of the same luminance as the striated pattern, were administered on $10 \%$ of the trials.

Gilinsky's results suggest that, with increased exposure to lines of a given orientation, the $S$ is able to discriminate less and less accurately lines of the same orientation but is able to discriminate with increasing accuracy lines running in a direction perpendicular to those just viewed. The first of these results can be readily accounted for in terms of the adaptation of retinal receptors, but the increasing sensitivity to lines oriented perpendicularly to the adapting grating cannot be so easily explained. On the basis of these two findings, Gilinsky interpreted her results as constituting empirical evidence for the existence of orientation-specific line detectors in the human visual system, similar to the ones discovered by Hubel and Wiesel in the cat and monkey.

As has been pointed out elsewhere (Woll, 1970), though, any Intcrpretation of psychophysical results in terms of such a higher-order hypothetical construct, even if it is a neurological construct with striking analogical support from direct physiological investigations with lower animals, requires that certain fundamental converging operations (Garner, Hake, \& Eriksen, 1956) be provided. Since Gilinsky's experiments fail to incorporate some of the most important of these, her results remain ambiguous and her interpretation of these results can be viewed as tenuous at best. The specific criticisms that can be raised against Gilinsky's methodology include the following:

(1) Percent correct, the measure of perceptual accuracy, is not an adequate indicator of perceptual performance when used in the context of an open-choice procedure in which Ss are to report the position of the target as horizontal or vertical. Such a measure takes account only of the S's "hit rate" (Egan \& Clarke, 1966; Swets, Tanner, \& Birdsall, 1961), or the probability of his giving an accurate report of the critical stimulus when it does in fact appear. No consideration is given by this measure to the S's "false-alarm rate," that is, to the probability of his reporting the presence (or, in this case, a given orientation) of the critical stimulus, even when it is not actually present. Thus, the use of a percent-correct measure in Gilinsky's experiment fails to rule out the confounding effects of the S's criterion for judgment of vertical and horizontal orientations upon his verbal report, from which report inferences about perceptual efficiency are made. This general criticism obviously applies to Gilinsky's threshold procedures, as well as to her procedure for the actual test sessions.

(2) The "catch trials" procedure does not provide an adequate indicator of the S's false-alarm rate (especially when the results of this procedure are not reported, as is the case in both Gilinsky papers), since it does not fumish sufficient information about the role of the S's criterion in the presence of the relevant stimuli (cf. Swets, 1961; Swets et al, 1961).

(3) Apart from employing an inadequate indicator methodology for finding the S's threshold, Gilinsky also chose an inappropriate level (i.e., $50 \%$ correct) at which to set her threshold criterion. Since, in a two-choice discrimination task, a correct report $50 \%$ of the time represents a purely chance level of responding, using this level of responding as a baseline makes it difficult to evaluate the effect of the experimental manipulation upon the S's ability to discriminate the orientation of the test grating.

(4) Since Gilinsky did not provide Ss with a fixation point, i.e., "S was free to scan the displays [1967, p. 395]," it seems likely that discrimination of both orientations might have been impaired by a "smearing effect" produced by the eye movements involved in scanning. A smearing explanation is also suggested by the fact that both the test and adapting gratings consisted of a "moiré" pattern, which produces an aftereffect attributed by MacKay (1961) "to the superposition of successive images as the eye moves... [which] appear as flickering shadows [p. 340]." MacKay also points out that these aftereffects "disappear if the retinal image is stabilized [p. 341] ." 
(5) It is also important to note that there was (essentially) no interstimulus interval (ISI) between presentations of the adapting and test gratings in Gilinsky's experiments. Eriksen's (1966) luminance-summation/contrast-reduction hypothesis suggests that under such conditions the luminances of the adapting and test patterns should sum maximally, thus creating a clearly discriminable checkerboard pattern in the case of perpendicularly oriented adapting and test patterns, and a virtually indiscriminable blurring for adapting and test patterns oriented in the same direction. Such an effect might account, at least in part, for Gilinsky's finding of slightly improved discrimination for perpendicularly oriented stimuli and slightly impaired discrimination for same-orientation test stimuli with increasing adapting durations.

In view of these methodological inadequacies, it follows that no single unequivocal interpretation of Gilinsky's findings can be made. Should these procedural flaws be corrected, moreover, any support for Gilinsky's interpretation in terms of masking of edge detectors to the exclusion of any more parsimonious explanation would seem to rest upon her finding of differential adaptation effects for the different adapting orientations. A number of investigators have called upon this finding of differential or cross adaptation to substantiate the existence of stimulus analyzing mechanisms (see Weisstein, 1969 for a review). As has been pointed out elsewhere (Woll, 1970), though, differential adaptation represents a necessary, but by no means sufficient, basis for drawing conclusions about neural detecting mechanisms.

In Gilinsky's case, however, these results take on a slightly different character, for, in her first experiment, not only was differential impairment of discrimination of same vs opposite orientation lines found, but also facilitation effects were found for two different conditions, namely, for short durations of the same-orientation adapting grating and for longer durations of opposite- (or perpendicular-) orientation adapting patterns. Whereas such facilitatory effects would seem to present problems for a simple adaptation or integration theory of masking, a more complex neural model, such as the ones formulated by Andrews (1965), by Sekuler and Pantle (1967), and by Weisstein (1968), could possibly handle such effects in terms of the notion of mutual or reciprocal inhibition of receptive fields. Thus, any confirmation of the existence of edge-detector mechanisms by Gilinsky's results requires that both differential inhibition and facilitation effects be found when the necessary converging operations have been made.

The experiment to be reported represents an attempt to investigate these "facilitation" and "inhibition" effects in the context of a more rigorous methodology. Specifically: (1) A two-alternative spatial forced-choice procedure was used instead of Gilinsky's open-choice procedure. In the context of a forced-choice methodology, percentage correct becomes a valid measure of perceptual efficiency because the effects of response bias are limited in a spatial forced-choice procedure to the negligible preference for the left or right side of the stimulus field (cf. Swets, 1961; Egan \& Clarke, 1966). (2) A 75\% threshold level was used instead of Gilinsky's 50\% level in order to, allow for a measurable and interpretable decrement and increment in performance. (3) A fixation point was provided in order to minimize any "smearing" that might result from excessive eye movements. (4) A lighted ISI was introduced between the adapting and test gratings in order to maintain the stimulus energy at a constant level while still minimizing the effects of contrast reduction. (5) Two different ISIs were used in order to be sure that a decrement in the discriminability of the test grating was not due simply to time-intensity reciprocity (cf. Kahneman \& Norman, 1964).

\section{Subjects}

\section{METHOD}

Four students (three graduate students and one undergraduate, one male and three female) served as paid Ss. All had normal or corrected-to-normal vision.

\section{Apparatus}

The major apparatus consisted of a three-field tachistoscope in which Sylvania F4T5/CWX lamps served as light sources; four Tektronix waveform generators, which controlled the duration for which each field was on; and four Tektronix pulse generators, which controlled the "off" time for each of the tachistoscopic fields.

The circular patterns presented in Field 1--the adapting stimuli-subtended an angle of $2 \mathrm{deg}$ and consisted of striated patterns of alternating black and white lines ("Moiré" Pattern 2, available from Edmund Scientific Co.), $1 \mathrm{~mm}$ wide, each subtending an angle of $2.44 \mathrm{~min}$ at the eye of the $S$, and oriented either vertically or horizontally. The test stimuli presented in Field 2, on the other hand, in keeping with the format of a forced-choice procedure, consisted of a nonstriate gray field and a striated pattern positioned side by side on the same stimulus card and matched in luminance by means of a Macbeth illuminometer. These two portions of the test stimuli were combined into four circular patterns, representing the four possible combinations of vertical and horizontal orientation and right and left position, and subtending $1.4 \mathrm{deg}$ of a visual angle. (This discrepancy in size between the adapting and test patterns was a feature of Gilinsky's experiments.)

Field 3, which served as both the fixation field and the ISI field, was lighted and contained a black fixation point in the center of the circular field, the fixation point subtending a visual angle of $50.4 \mathrm{~min}$ and the circular field an angle of $2 \mathrm{deg}$. All three stimuli were located at viewing distances of $59 \mathrm{in}$. in their respective fields, were front-lighted, and their respective luminances were adjusted to $1.4,1.4$, and $1.8 \mathrm{~mL}$.

\section{Procedure}

Ss were first run for a threshold session, in which the offset of the lighted fixation field was followed by the onset of the test pattern. The luminance of the fixation field had, as in the Gilinsky experiment, been matched as closely as possible with the luminance of the adapting field. The test patterns were presented during this threshold session in blocks of horizontal and vertical orientation in counterbalanced order, with the S's response alternatives on each trial being limited to "left" or "right." The original plan was to determine for each $S$ the duration of the test stimulus at which he judged correctly on $75 \%$ of the trials the side on which the striated half occurred. Since Gilinsky (1967, 1968) reported that she found little difference between Ss' thresholds for the vertical and horizontal orientations, little difficulty was anticipated in this regard.

As it turned out, however, all Ss showed at least a 1 -msec difference between their thresholds for the two orientations; this small temporal difference between the two thresholds made a fairly large difference in the Ss' accuracy for the two orientations (e.g., see Kahneman, 1968). Thus, although none of the four Ss showed a difference in thresholds of greater than $3 \mathrm{msec}$, the duration at which each $S$ achieved $75 \%-80 \%$ accuracy for the less discriminable orientation nevertheless proved also to be the duration at which he achieved 95\%-100\% accuracy for the more discriminable orientation. Furthermore, whereas for three out of the four Ss the vertical orientation proved easier to discriminate, for the fourth $S$ the horizontal orientation elicited better performance.

The discrepancy between the thresholds for vertical and horizontal orientations turned out to be a fortunate situation, at 
least in one respect: In the course of the test sessions, it became apparent that the degradation of the test stimulus by the adapting pattern was so great that even the $75 \%-80 \%$ threshold was not at a high enough level to give clearcut results. (The determined thresholds for the four Ss ranged from 4 to $13 \mathrm{msec}$, with the size of the discrepancies between the two thresholds paralleling the rank order of the Ss with regard to their determined thresholds; that is, the $S$ with a 4-msec threshold showed a 1-msec discrepancy, while the $S$ with a $13-\mathrm{msec}$ threshold showed a 3-msec discrepancy.) The importance of this differential threshold factor and of the difference in orientation bias among $S s$ will become clear in the analyses reported below.

The procedure for each of the test sessions was as follows: On each trial the $\mathrm{S}$ was to focus his attention on the fixation point in the center of Field 3. When this fixation point was clearly in focus, the $S$ was to trigger the onset of the adapting pattern, which remained on for $500,1,000$, or 3,200 msec. $^{2}$ The offset of this adapting pattern was followed immediately by the reappearance of the fixation point and light surround, which remained on for a lighted ISI of either 320 or $630 \mathrm{msec}$. The offset of the ISI was immediately followed by the test pattern, which remained on for the S's threshold duration and, in turn, was immediately followed by the fixation field.

The experimental variables manipulated, then, were the following: (1) The duration of the adapting pattern (AD) was set at $500,1,000$, or $3,200 \mathrm{msec}$, with the order of these $\mathrm{AD}$ conditions being arranged within each session in a randomized blocks design; (2) the orientation of the adapting pattern (i.e., vertical or horizontal) was randomized across trials; (3) the ISI was set at either 320 or $630 \mathrm{msec}$, with the order of these ISI durations being counterbalanced across . experimental sessions; and (4) the orientation of the striated portion of the test stimulus (i.e., vertical or horizontal) and the position of this striated portion (i.e., right or left) were both randomized across trials independently of the orientation of the adapting pattern. Eight test sessions were conducted for each $S$, and each $S$ was run under each of the 24 combinations of stimulus conditions, yielding a mean of 192 observations per combination.

\section{RESULTS}

The percentage of correct responses was first analyzed in a five-way analysis of variance $[A D$, ISI, adapting-field orientation, test-field orientation (same or different with respect to the adapting field), and Ss]. The results of this analysis

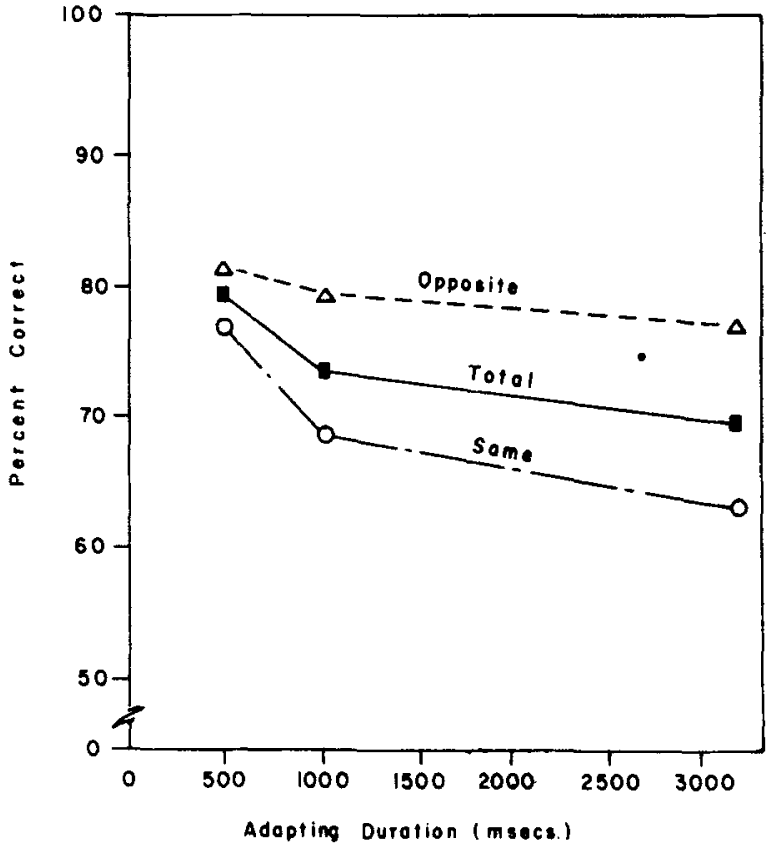

Fig. 1. Percent correct for same-vs opposite-orientation stimuli and totals as a function of adapting duration.

showed three significant main effects $[A D$, $F(2,6)=15.69, p<.005$; test orientation, $\mathrm{F}(1,3)=55.80, \quad \mathrm{p}<.005 ;$ and Ss, $F(3,6)=251.61, \quad p<.001]$ and 17 significant interaction effects, with the latter reflecting mainly Ss effects.

Now, in view of the relatively large range of threshold values reported earlier and the differences among $S s$ in orientational "preference," the significant overall Ss effect is not an unexpected result. However, these same considerations also suggest that many, if not all, of the significant interaction effects involving a Ss term are spurious-at least in one important sense. For although, as was indicated above, none of the four $\mathrm{Ss}$ in this experiment showed a horizontal-vertical threshold discrepancy of over $3 \mathrm{msec}$, what appears to be more important for interpreting the significance of the above interaction effects is the fact that, whereas three of the four Ss showed a lower threshold for the vertical than for the horizontal orientation, the fourth $\mathrm{S}$ showed a lower horizontal threshold. In other words, a crossover effect occurred.

Although a thorough examination of this fourth S's performance suggests that he did not differ greatly from the other Ss with respect to the overall effects of the experimental manipulations (i.e., adapting duration, ISI, and adapting orientation), in the above analysis much of the variance from his performance was (erroneously) assigned to the above interaction effects. Thus, a modification of the above analysis was undertaken, which consisted essentially of reversing this fourth S's orientational bias and thereby substituting the factor of high- vs low-threshold orientation for the adapting orientation dichotomy. The purpose of this reanalysis, then, was to compare statistically Ss' performance on stimulus patterns that the threshold sessions had shown to elicit comparable performances, rather than on stimuli judged as objectively comparable.

Such a reanalysis did indeed provide a clearer and more realistic picture of the factors operating in this experiment. In the first place, four significant main effects were found: $\operatorname{AD}, F(2,6)=15.49, p<.005$; high vs low test-threshold orientation, $\mathrm{F}(1,3)=135.68, \mathrm{p}<.005 ;$ adapting orientation (same vs opposite with respect to test orientation), $F(1,3)=55.80$, $p<.005$; and Ss, $F(3,6)=6.34, p<.05$. The most important result of this reanalysis, though, is the fact that all of the previously significant Ss' interaction effects disappeared (i.e., all were found to be statistically nonsignificant), and the only four significant interactions that remained were $\mathrm{AD}$ by Adapting Orientation $[F(2,6)=9.37, p<.025]$, High vs Low Test-Threshold Orientation by Adapting Orientation $[F(2,6)=13.47, \mathrm{p}<.05]$, ISI by High vs Low Test-Threshold Orientation $[F(2,6)=11.61, p<.01]$, and ISI by High vs Low Threshold by Adapting Orientation $[F(1,3)=20.13, p<.025]$

The interpretation of these two analyses is necessarily a complex one; therefore, it seems advisable to begin by examining the similarities or constancies between the two 


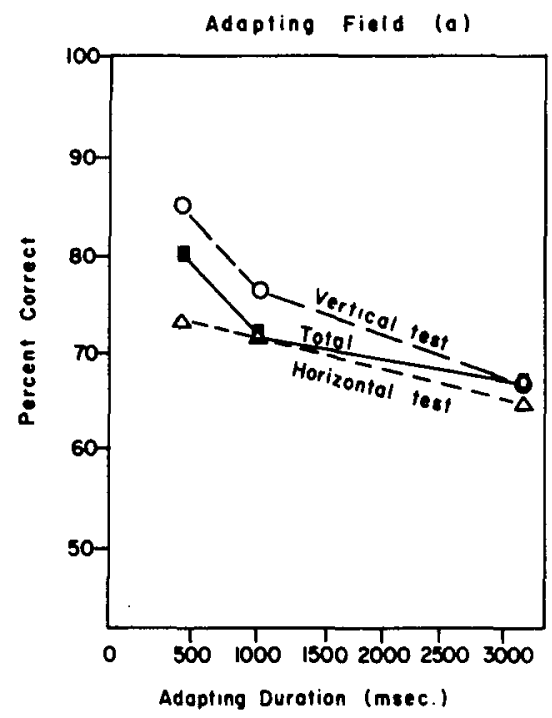

Fig. 2. Percent correct for vertical and horizontal test gratings and totals as a function of adapting duration, plotted separately for vertical and horizontal adapting orientations.

analyses. First of all, it is clear from both analyses that adapting duration (AD) had a significant effect on the discriminability of the test gratings for all Ss. Furthermore, it is not surprising that both analyses show (despite the different labels used) that the test grating is significantly less discriminable when viewed following an adapting pattern of the same orientation than when viewed after an adapting pattern of the opposite orientation. The finding that is of particular interest, though, is the $\mathrm{AD}$ by Same vs Opposite Orientation interaction, which is plotted in Fig. 1 or, more specifically, the two aspects of this interaction that are critical to Gilinsky's demonstration of the adaptation of neural edge detectors. Although the overall interaction effect is statistically significant in both analyses-a result that is predicted by both a simple aftereffects interpretation and Gilinsky's more complex formulation-a glance at Fig. 1 indicates that there is no evidence of the facilitatory effects found by Gilinsky in her experiments.

It might be argued, of course, that Fig. 1 is not comparable to Gilinsky's findings, because it does not present the results for the vertical and horizontal adapting orientations separately. Replotting the results in this way does lead to a very different picture, as is clear from Fig. 2. It should also be apparent from Fig. 2, however, that such a presentation of the data is somewhat misleading. Once again, this is because the results plotted in Fig. 2 do not reflect the differential effect of different adapting orientations as much as they do the simple confounding effect of
Horizontal

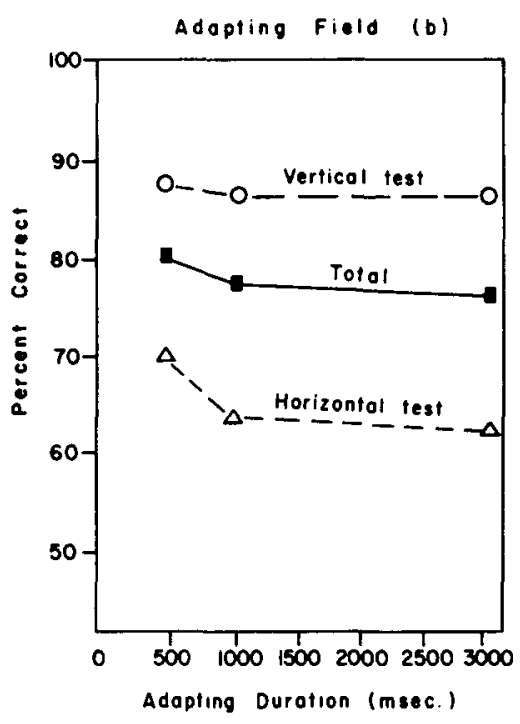

the test-pattern orientation. In other words, same-orientation test stimuli are discriminated more accurately after a vertical adapting stimulus than are gratings of the opposite orientation, simply because the "same-orientation" stimuli in this instance are vertical lines, which are seen more clearly than are horizontal lines under all experimental conditions (at least for three of the four Ss). Similarly, the effects for the horizontal adapting condition can be explained by the fact that the converse set of relations holds.

The upshot of the foregoing discussion appears to be that it is the "preferred" orientation of the test field for individual Ss that constitutes the most powerful factor in the present study. Although this variable of high vs low test threshold controls only $4 \%$ of the variance in the second analysis, the rather striking difference in the results of the first and second analyses makes it clear that threshold orientation is indeed a relatively potent variable.

Perhaps an additional word is in order about the significant interaction effect found in the second analysis between ISI, high vs low test threshold, and adapting orientation. As pointed out earlier, two different ISI durations were included in order to make sure that decrements in discrimination of the test gratings were not attributable simply to the effects of time-intensity reciprocity. It might be added here that the long ISI also allowed for greater recovery of retinal cells from exposure to the adapting field and, thus, better discrimination of the test stimulus. Both of these effects contribute to the results shown in Table 1. As these figures make clear, the primary source of the significant interaction effect is the much greater accuracy on the same adapting orientation-low test threshold trials at the longer ISI duration than at the shorter one (Column 1 of Table 1).

\section{DISCUSSION}

The results of this experiment, although by no means unequivocal in themselves, do cast some doubt upon Gilinsky's findings, and do suggest that the results from her general paradigm can be explained in less exotic terms than she has used. The decrement in discriminative performance with greater adapting exposures in the present experiment can be parsimoniously explained in terms of interference by "complementary" afterimages, or, more specifically, by the adaptation of retinal cells that have just been stimulated by the adapting pattern. Such an explanation is supported by anecdotal evidence from the Ss in this experiment, several of whom commented that the greatest difficulty in the experimental task was deciding whether they were basing their judgments of position on the second stimulus or on the aftereffects of the first. This interference by afterimages, of course, constituted a much greater problem when the adapting stimulus was of the same orientation as the test stimulus, a fact which was also reflected in Ss' informal comments on the experiment.

Although the difference found between the accuracy of discrimination for same and opposite orientations was surprisingly large, in retrospect, the size of this difference should not have been that surprising. Studies by Campbell and Kulikowski (1966), by Sekuler (1965), and by Houlihan and Sekuler (1968) all suggest that the interfering effects of a prior stimulus decrease as the angular difference

Table 1

Percent Correct for the Two Different ISIs, High Vs Low Test Threshold, and Same Vs Different Adapting Orientation

\begin{tabular}{|c|c|c|c|c|}
\hline \multirow[b]{2}{*}{ ISI } & \multicolumn{2}{|c|}{ Low Test Threshold } & \multicolumn{2}{|c|}{ High Test Threshold } \\
\hline & $\begin{array}{c}\text { Same } \\
\text { Adapting } \\
\text { Orientation }\end{array}$ & $\begin{array}{c}\text { Opposite } \\
\text { Adapting } \\
\text { Orientation }\end{array}$ & $\begin{array}{c}\text { Same } \\
\text { Adapting } \\
\text { Orientation }\end{array}$ & $\begin{array}{c}\text { Opposite } \\
\text { Adapting } \\
\text { Orientation }\end{array}$ \\
\hline $320 \mathrm{Msec}$ & $74 \%$ & $98 \%$ & $58 \%$ & $61 \%$ \\
\hline $630 \mathrm{Msec}$ & $92 \%$ & $97 \%$ & $60 \%$ & $63 \%$ \\
\hline
\end{tabular}



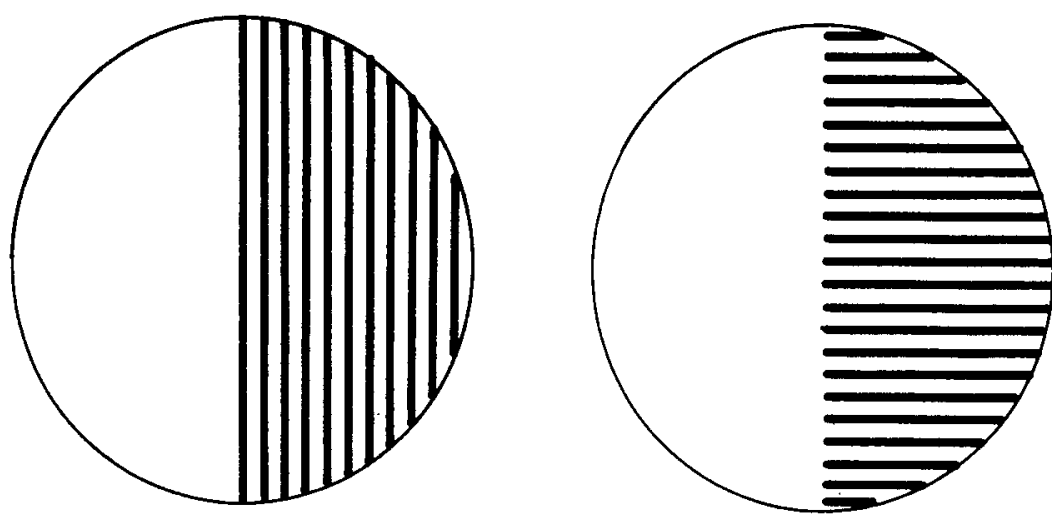

Fig. 3. Examples of test stimuli.

in orientation increases. It would be smallest when the angle between the two stimuli is greatest. In the case of straight lines, of course, the largest possible angle is $90 \mathrm{deg}$. In addition to sheer perceptual differences, there is the possibility that the greater discriminability of the oppositely oriented stimuli may have allowed a practice effect to operate to further increase performance for this condition as Ss "learned" how to make use of the greater number of cues available to them under these circumstances. Schiller (1965), for example, has observed that "Repeated trials with patterns as masking stimuli lead to a decrement in the masking effect ... [which] suggests complex processes of perception in which learning might play a role [p. 198]." This possibility is supported by the comments of at least one $S$, who reported that he was able to discriminate the opposite orientation test stimuli better as the experiment progressed.

The finding of a differential threshold for vertical and horizontal gratings, however, raises a problem. One explanation of this finding, which is consistent with the reasoning of Gilinsky and others (cf. Weisstein, 1969), is that the differential thresholds for the two orientations must be attributable to some sort of differential detecting mechanism in the human visual system. Such a conclusion, however, would not only be inconsistent with the findings of several studies (e.g., Campbell \& Kulikowski, 1966; Taylor, 1963) reporting that there is little if any difference in discriminability of these two orientations, but would also be unparsimonious. A more plausible and easily testable explanation ${ }^{3}$ in the context of the present experiment is that a spatial forced-choice procedure in which vertically and horizontally striated patterns are used as stimuli and in which the S's view of these stimuli is limited by a circular aperture automatically gives the advantage to the vertical pattern.
Inspection of the forced-choice situations illustrated in Fig. 3 suggests at least one of the reasons why the vertical pattern is at an automatic advantage. As these examples point out, a vertical line extending from the top to the bottom of a circle serves to divide the circle cleanly into two parts, whereas a series of alternating black and white horizontal lines extending halfway across the circle does not make such a clean division. (This point can perhaps be better expressed in terms of luminance contrast between the white line on one side of the dividing line and the solid gray area on the other, creating in effect, a figure-ground relationship.) In addition, prolonged viewing of the horizontal lines results in their apparent extension into the blank, nonstriate area, whereas the result for the vertical lines is an apparent extension outside the top and bottom edges of the circle.

One way of "testing" this explanation would be to set up a spatial forced-choice situation in which $\mathrm{S}$ is required to choose between the top and bottom halves of this same circle, using these same stimuli. In this situation, one would expect vertical lines to be discriminated less readily since it is now the horizontal lines that cleanly bisect the circle and, further, it is the vertical lines that are subject to the "illusion" mentioned above. Such a converging operation does not, of course, explain or eliminate the results of the one $S$ who discriminated horizontal lines more clearly (although it would be particularly interesting in his case to see if he continued, as would be predicted, to see horizontal lines more easily). The results for this $S$, however, are probably best viewed as an individual difference variable rather than as illustrating some basic, general psychological principle; and, indeed, the very presence of this one exception to the rule (if one can derive a general rule from such a small samplc) argues against any explanation in terms of some sensitive detecting mechanism common to all humans.

\section{REFERENCES}

ANDREWS, D. P. Perception of contours in the central fovea. Nature, 1965, 205, 1218-1220.

CAMPBELL, F. W., \& KULIKOWSKI, J. J. Orientational selectivity of the human visual system. Journal of Physiology, 1966, 187, 437-455.

EGAN, J. P., \& CLARKE, F. R. Psychophysics and signal detection. In B. J. Sidowski (Ed.), Experimental methods and instrumentation in psychology. New York: McGraw-Hill, 1966. Pp. 211-246.

ERIKSEN, C. W. Temporal luminance summation effects in forward and backward masking. Perception \& Psychophysics, 1966, 1, 87-92.

ERIKSEN, C. W., \& HOFFMAN, M. Form recognition at brief durations as a function of adapting field and interval between stimulations. Joumal of Experimental Psychology, 1963, 66, 485-499.

GARNER, W. R., HAKE, H. W., \& ERIKSEN, C. W. Operationism and the concept of perception. Psychological Review, 1956, 63, 149-159.

GILINSKY, A. Masking of contour-detectors in the human visual system. Psychonomic Science, 1967, 8, 395-396.

GILINSKY, A. Orientation-specific effects of patterns of adapting light on visual acuity. Journal of the Optical Society of America, $1968,58,13-18$.

HOULIHAN, K., \& SEKULER, R, W. Contour interactions in visual masking. Journal of Experimental Psychology, 1968, 77, 281-285.

HUBEL, D. H., \& WIESEL, T. N. Receptive fields of single neurons in the cat's striate cortex. Journal of Physiology, 1959, 148, 574-591.

HUBEL, D. H., \& WIESEL, T. N. Receptive fields and functional architecture of monkey striate cortex. Journal of Physiology, 1968, 195, 215-245.

KAHNEMAN, D. Method, findings, and theory in studies of visual masking. Psychological Bulletin, 1968, 70, 404-425.

KAHNEMAN, D., \& NORMAN, J. The time-intensity relation in visual perception as a function of observer's task. Journal of Experimental Psychology, 1964, 68, 215-220.

MacKAY, D. M. Interactive processes in visual perception. In W. A. Rosenblith (Ed.), Sensory communication. Cambridge: M.I.T. Press, 1961. Pp. 339-355.

NEISSER, U. Cognitive psychology. New York: Appleton-Century-Crofts, 1967.

SCHILLER, P. H. Monoptic and dichoptic visual masking by patterns and flashes. Journal of Experimental Psychology, 1965, 69, 193-199.

SEKULER, R. W. Spatial and temporal determinants of visual backward masking. Journal of Experimental Psychology, 1965, 70, 401-406.

SEKULER, R. W., \& PANTLE, A. A model for after-effects of seen movement. Vision Research, 1967, 7, 427-439.

SWETS, J. A. Decision theory and psychophysics: A review. Psychometrika, 1961, 26, 49-63.

SWETS, J. A., TANNER, W. P., \& BIRDSALL, T. G. Decision processes in perception. Psychological Review, 1961, 68, 301-320.

TAYLOR, M, M. Vertical discrimination and orientation. Journal of the Optical Society of America, 1963, 53, 763-765.

WEISSTEIN, N. A Rashevsky-Landahl neural net: Simulation of metacontrast. Psychological Review, 1968, 75, 494-521.

WEISSTEIN, N. What the frog's eye tells the human brain: Single cell analyzers in the human visual system. Psychological Bulletin, 1969, 72, 157-176.

WOLL, S. B. Psychophysical studies of 
edge-detector mechanisms in the human visual system. Unpublished Master's thesis, University of Illinois, 1970.

\section{NOTES}

1. The procedure to be described was employed in the first of the two experiments reported by Gilinsky. The procedure followed in the second study (1968) was basically the same as the one to be described, except that oblique lines ( 45 and 135 degrees) were used in addition to vertical and horizontal ones, and the primary dependent measure in the second experiment was threshold duration rather than percent correct. The criticisms to be raised here against the first experimental procedure also apply to the second procedure.

2. The values of 320,630 , and $3,200 \mathrm{msec}$ were used instead of 300,600 , and $3,000 \mathrm{msec}$ because of the constraints imposed by the
Tektronix equipment.

3. This general explanation and the following proposal for testing it were worked out in collaboration with Steve Leeb.

(Accepted for publication July 12, 1969.) 